\title{
Prevalence and Risk Profile of Unread Messages To Patients In A Patient Web Portal
}

B.H. Crotty ${ }^{1,2}$; A. Mostaghimi2,3; J. O'Brien'; A. Bajracharya' ${ }^{1,2}$; C. Safran ${ }^{1,2}$; B.E. Landon ${ }^{2,4}$

${ }^{1}$ Division of Clinical Informatics, Beth Israel Deaconess Medical Center, Boston, MA; 2. Harvard Medical School, Boston, MA; ${ }^{3}$ Department of Dermatology, Brigham \& Women's Hospital, Boston MA; ${ }^{4}$ Department of Health Care Policy, Harvard Medical School, Boston, MA

\begin{abstract}
Keywords
Personal health records, email, communication, secure messaging, electronic health records

\section{Summary}

Background: Excitement around the adoption of electronic communication between physicians and patients is tempered by the possibility of increased clinical and legal risk. If patients do not read messages in a timely fashion, duplicative communication efforts may be required and patient safety may be jeopardized.

Objective: We sought to assess the prevalence and risk profile of unread messages in a mature patient portal.

Methods: We analyzed six years of messages (2005-2010) from physicians to patients to determine the prevalence and associated characteristics of unread messages in a patient portal. We focused on clinical messages, and excluded announcements. Because some physicians sent clinical messages to groups of patients, we labeled messages sent to more than 5 patients as "outreach" messages and excluded them from general analyses. We performed a chart review of 75 clinical messages to assess for harm.

Results: We found that $3 \%$ of clinical messages were unread after 21 days. Messages arriving outside of business hours were slightly more likely to go unread (RR $1.1595 \%$ Cl 1.11-1.19). Patients who were male (OR $1.14 \mathrm{Cl} 1.04-1.26)$ African American (OR $1.69 \mathrm{Cl} 1.29-2.22$ ) or Hispanic (OR $1.74 \mathrm{Cl} 1.17-2.59$ ), or in the lowest income group (OR $1.72 \mathrm{Cl} 1.19-2.49$ ) were more likely to have unread messages. Chart review showed no evidence of harm, but $13 \%$ of sampled unread messages were associated with potential delays in care. Incidentally, we found $50 \%$ of the physicianinitiated outreach messages were unread.

Conclusions: Overall, secure messaging appears a safe form of communication, but systems to notify senders when messages are unread may have value. While most clinical messages were read, many outreach messages were not, providing caution for relying on such systems for information dissemination. Similar to other studies, differences by race and income were observed and require further study.
\end{abstract}

\section{Correspondence to:}

Bradley H Crotty MD MPH

Division of Clinical Informatics

1330 Beacon Street, Suite 400

Brookline MA 02446

Email: bcrotty@mail.harvard.edu

\author{
Appl Clin Inform 2015; 6: 375-382 \\ http://dx.doi.org/10.4338/ACl-2015-01-CR-0006 \\ received: January 12,2015 \\ accepted: April 17, 2015 \\ published: June 12, 2015 \\ Citation: Crotty BH, Mostaghimi A, O'Brien J, Bajra- \\ charya A, Safran C, Landon B. Prevalence and Risk Pro- \\ file Of Unread Messages To Patients In A Patient Web \\ Portal. Appl Clin Inf 2015; 6: 375-382 \\ http://dx.doi.org/10.4338/ACI-2015-01-CR-0006
}




\section{Introduction}

Patients and medical providers are increasingly using patient portals to exchange secure electronic messages and facilitate clinical care $[1,2]$. Patient send messages to ask questions, coordinate care such as appointments, obtain referrals or prescriptions, provide an update to their clinician, or inquire about a new symptom [3-5]. Guidelines for electronic patient-clinician communication have stated that such messaging should be used for non-urgent communication, and encourage patients to acknowledge receipt of messages [6]. Studies have shown that less than $3 \%$ of messages that patients send to clinicians are thought to be time-sensitive $[7,8]$.

In the era of accountable care organizations, secure electronic messaging offers providers opportunities to communicate with patients to promote preventative health services, coordinate care, communicate test results and reply to patient concerns [9] [10]. Patient portals and secure messaging can supplement the work of clinicians and care managers. However, messages that are not read by patients may reduce the reliability of these systems, resulting in clinical risk for patients and legal risk for physicians [7]. Physician response times and factors for delayed reading of messages have previously been reported, but little is known about patients' time to read messages and rate of unread messages [11].

In this report, we set out to assess how often messages sent by physicians to patients go unread in a patient portal. Additionally, we sought to determine patient and message level factors associated with unread messages, and to assess for possible adverse events resulting from unread messages.

\section{Methods}

\section{Setting and Platform}

PatientSite, in operation since 2000 at Beth Israel Deaconess Medical Center (Boston, MA), is an integrated personal health record providing patients a view of their electronic data and the ability to electronically communicate with their providers $[12,13]$. PatientSite has a secure messaging architecture that requires patients and clinicians to log into the platform in order to read and send messages. When a new message is available, the recipient receives an email describing that they have new activity in their account, and prompts them to log into the portal. These notification messages are structured similarly for these different announcements, with the type of message (clinical, broadcast/announcement, support, or reminder) noted in the body of the email. The sender's identity is not identified in the email to maintain privacy.

Through December 31, 2010, 272 attending physicians were registered on PatientSite, and 49,778 patients $(22.7 \%$ of health system patients seen in that year) were enrolled in the portal. The PatientSite population has historically been less diverse than the population served by the Medical Center, which includes Boston and surrounding communities in eastern Massachusetts.[13] As of 9/2010, the median age of PatientSite users at their time of enrollment was 44 (Interquartile Range 32-55), $62 \%$ were women, $73 \%$ were white, and $87 \%$ were privately insured.

\section{Study Design}

We assessed the prevalence of unread messages, if message timing or patient characteristics impacted whether messages went unread, and, in a sample, whether unread messages presented any potential for clinical harm. We retrospectively analyzed secure messages from 2005-2010, after allowing for a lead-in period from 2000-2004. Duplicate messages were removed.

We focused only on "clinical" messages sent from attending physicians to patients and excluded messages labeled as "broadcast" which denote announcements for groups of patients or an entire practice. We found that some clinicians directly contacted groups of patients through messages labeled as "clinical." We characterized such messages as "outreach" messages if they were addressed to more than 5 recipients and excluded them from our general analyses. We allowed up to five recipients to cover scenarios where family members who care for a patient may be copied on messages. 
We determined the prevalence and general characteristics of unread messages, and defined unread messages as those not read within 21 days. In sensitivity analyses, we also examined cutpoints of 3 and 7 days. We then assessed whether a message going unread was related to it being sent during business hours (8am-5pm) or off hours (including weekends) using Fisher's exact test.

To assess for differences in the prevalence of unread messages according to sociodemographic characteristics, we performed multilevel logistic regression, clustering by patient. Variables of interest included age, sex, self-reported race as stored in the clinical information system, and socioeconomic status using imputed household income based on census block data from the American Community Survey 2010[15]. Because some of these variables could change over the course of the study, we analyzed only a cross-section of data from 2010, the last year of our sample. All analyses were run on SAS v 9.3 (SAS Institute, Cary, NC).

To assess for potential clinical harm resulting from a patient not reading a message from a physician, two investigators (BC, JO) performed a chart review of 75 message threads and related medical records from a random sample of 75 unread clinical messages. Specifically, we identified outpatient, emergency department, and hospital use within 30 days of an unread message. We also identified other forms of documented communication in the medical record, such as letters or phone calls. We categorized the messages by content type and as having caused or having potential to cause harm, such as a delay in care or other adverse event. We categorized delays and adverse events as "potential" if messages had important information requiring an action by the patient where no other redundant communication modalities (i.e. phone call) occurred, and "definite" if it resulted in a delay, adverse event, or emergency visit as assessed by chart review. Differences were adjudicated by a third investigator $(\mathrm{AB})$ and then by consensus.

The Institutional Review Board of Beth Israel Deaconess Medical Center approved the study.

\section{Results}

Excluding outreach messages, the rate of unread messages for patients was $3.1 \%$ at 21 days. ( $>$ Table 1) For messages that were read, the median read time was 3 hours (interquartile range 1-15 hours). Messages sent outside of business hours (weekdays $8 \mathrm{am}-5 \mathrm{pm}$ ) were slightly more likely to go unread (Relative Risk 1.15 and 95\% Confidence Interval 1.11-1.19). After adjustment using multivariable regression, patients who were male, African American, Hispanic, and with annual household income less than $\$ 25,000$ were statistically more likely to have unread messages ( Table 2).

Inclusive of outreach messages, $16.6 \%$ of messages were unread at 21 days. Sixteen physicians sent 1,219 distinct messages to multiple patients, generating 158,577 outreach messages. These messages accounted for $29 \%$ of the sample, and half of these were unread.

\section{Chart Review And Potential For Harm}

Of the unread messages we manually reviewed, $31 \%$ were initiated by the doctor while $69 \%$ were replies to a patient message. The subject line encapsulated the information entirely in 8 messages (11\%), meaning that the message body did not contain any additional information. Physicians also contacted the patient through a call or letter in $18 \%$, the majority of which were for results reporting and management. ( Table 3) No patients were harmed from missed messages, though 10 messages (13\%) were associated with a potential delay of care (e.g. reminder for endoscopy) and 3 messages (4\%) were associated with the potential for an adverse event (communication of an abnormal laboratory value, need for follow-up action, scheduling a visit for a high risk symptom).

\section{Discussion}

In this retrospective study of secure messaging, we found that patients read over $90 \%$ of clinical messages within 3 days. Given the asynchronous nature of secure messaging, these findings show that messages sent from physicians to patients are reliably read in a timely manner. Unread messages were relatively rare events, and we found no instances of harm to patients from unread messages. 
However, we did find that an estimated $13 \%$ of unread messages were associated with a potential delay in care [11].

Incidentally, we found that about half of outreach messages went unread. While these were only 1,219 messages sent by sixteen clinicians, they did account for nearly a third of all clinical messages received by patients. These results suggest that secure messaging can serve an important function for patients and doctors, but may have limitations for prospective, population-based outreach that require further exploration. It may be possible that patients are less likely to read messages that arrive to them "unexpectedly," meaning not from their doctor near in time to an episode of care. However, patients may have simply deleted these messages from their inboxes without reading them based upon the subject line.

We did find differences in unread messages according sociodemographic factors, including race/ ethnicity and income. We found that patients who were African American, Hispanic, or with household incomes $<\$ 25,000$ had a slightly higher odds of having unread messages. It will be important to further explore this finding, and to understand the implications of technology use across different groups of patients, particularly since these were all enrollees who had affirmatively signed up for the system and had access to a computer and the internet. A previous study by Yamin et. al. had shown that there were differences in portal enrollment by race and income, but that once enrolled, utilization and messaging did not differ by race, and only differed in the lowest income quartile with their association with lower messaging [14]. Analysis of unread messages adds additional information, and suggests that differences are small but present with respect to age, sex, and income.

Why might patients enroll in a portal that offers secure messaging with clinicians but not read messages? A few possibilities exist, including factors related to Shannon's communication theory namely the signal to noise ratio in the portal and in the traditional email inbox - as well as factors specific to the portal and communication preferences of the patient $[16,17]$. Patients may unintentionally perceive notification emails as duplicates, perceive them to be noise in the system such as appointment reminders or announcements, or miss them altogether in their inbox. Additionally, if patients change their email addresses, notification messages may bounce but not alert the sender. Or, if they have not accessed the system for some time, they might have lost their account information.

Curiously, messages sent by physicians during working hours were more likely to be read at 21 days. In line with the above discussion, it may be that messages sent off hours may be perceived as noise more often, rather than an important message from their clinician.

For practices and vendors providing patients portals, our study offers several lessons. Secure messaging appears to be a relatively safe communication strategy, but could be made better with tracking, message read receipts, and message escalation for unread messages past a certain time threshold [18]. For some busy patients, reaching them by email may actually be timelier than telephone calls, and categorizing messages by importance (read by end of day, read by end of week) may be helpful to alert patients to the importance of the message.

Standardizing workflows and communication practices within clinics, recording patients' communication preferences, and setting expectations may help clinicians and patients use secure messaging more efficiently. Our chart review found that many providers were duplicating work, doing both email and mailing hard copies such as for the communication of laboratory and imaging results. Tracking and escalation of unread messages, with back-ups of mailed copies and/or phone calls by staff, may streamline this process. Indeed, considering secure messaging as a core communication element in practice workflow may be useful to all parties [19].

For practices adopting the tools for population-based outreach [20,21], many messages were still read, and indeed patients may wish to be alerted to news and care reminders in this fashion. Potentially, outreach may be improved if notification emails can clearly reflect the type of message (e.g. "care reminder" or "message from your doctor") where possible and where privacy is not compromised. Again, capturing communication preferences of patients is likely to be helpful.

Our study should be interpreted within the context of the following limitations. This is a single institution study, but one of a mature patient portal deployed to over 70 practices. For our quantitative analysis, we did not have access to the ambulatory clinical data to adjust for comorbidities, and it is possible that adjustment might explain variation seen across different groups of patients. Finally, the chart review portion of our study had limited power to detect harm from relatively rare events. 
In summary, secure messaging appears to be a reliable way for physicians to communicate with patients, but may have limitations when used for outreach messaging. While we observed no adverse events from unread messages in our sample, we did observe delays in recommended testing, referrals, and treatment. A minority of these delays in communication had the potential to lead to adverse events. Features for redundancy and closed-loop communication appear necessary.

\section{Clinical Relevance}

Patients read $91 \%$ of secure messages within 3 days, and $97 \%$ within 21 days. In this study, no adverse events were reported from patients not reading messages, but delays in care were possible when messages were not read in a timely fashion. Systems should be designed to notify practitioners and their staff when messages go unread.

\section{Acknowledgements}

The authors report no conflicts of interest. Drs. Crotty, Mostaghimi, Safran, and Landon conceived of the study design. Drs. Bajracharya, Crotty, and Mrs. O’Brien assisted with data analysis and review. All authors critically reviewed the manuscript and approved the final version. Dr. Crotty was supported by an Institutional National Research Service Award (No. T32HP12706) and by the Ryoichi Sasakawa Fellowship Fund. Dr Bajracharya is supported by the National Library of Medicine grant T15LM007092.

\section{Human Subjects Protection}

This study was approved by the Beth Israel Deaconess Medical Center's Institutional Review Board 
Table 1 Summary statistics for clinical messages and outreach messages (secure messages sent by physicians to five or more recipients, but not labeled as announcements).

\begin{tabular}{|c|c|c|c|c|}
\hline & & Clinical Messages & $\begin{array}{l}\text { Outreach Clinical } \\
\text { Messages }\end{array}$ & Overall \\
\hline \multicolumn{2}{|c|}{ Number of Messages 2005-2010 } & $388,911(71.0 \%)$ & $158,577^{\dagger}(29.0 \%)$ & $547,488(100 \%)$ \\
\hline \multicolumn{2}{|c|}{$\begin{array}{l}\text { Median Read Time (Interquartile Range) } \\
\text { Excluding 70,546 messages never read }\end{array}$} & $3(1-15)$ hours & $20(4-102)$ hours & $5(1-20)$ hours \\
\hline \multirow[t]{3}{*}{ Percent Unread } & 3 days & $9.1 \%$ & $61.0 \%$ & $24.2 \%$ \\
\hline & 7 days & $5.1 \%$ & $54.5 \%$ & $19.4 \%$ \\
\hline & 21 days & $3.1 \%$ & $49.8 \%$ & $16.6 \%$ \\
\hline
\end{tabular}

${ }^{\dagger}$ Sixteen physicians sent 1219 unique messages to multiple recipients, accounting for a total 158,577 delivered messages.

\begin{tabular}{|c|c|c|c|c|}
\hline & & Odds Ratio & $95 \% \mathrm{Cl}$ & $P$ value \\
\hline \multicolumn{2}{|l|}{ Male Sex } & 1.14 & $1.04-1.26$ & $P<0.01$ * \\
\hline \multirow[t]{4}{*}{ Race } & White & Ref & - & - \\
\hline & Black & 1.69 & $1.29-2.22$ & $P<0.01$ * \\
\hline & Asian & 1.01 & $0.77-1.32$ & $P=0.96$ \\
\hline & Hispanic & 1.74 & $1.17-2.59$ & $\mathrm{P}<0.01$ * \\
\hline \multirow[t]{5}{*}{ Age } & $18-34$ & Ref & - & - \\
\hline & $35-54$ & 1.13 & $0.99-1.30$ & $P=0.07$ \\
\hline & $55-64$ & 0.96 & $0.82-1.11$ & $P=0.56$ \\
\hline & $65-74$ & 0.94 & $0.77-1.14$ & $P=0.50$ \\
\hline & $\geq 75$ & 1.16 & $0.87-1.53$ & $P=0.31$ \\
\hline \multirow{4}{*}{$\begin{array}{l}\text { Annual } \\
\text { Houshold } \\
\text { Income }\end{array}$} & $<\$ 24,999$ & 1.72 & $1.19-2.49$ & $P<0.01^{*}$ \\
\hline & $\$ 25,000-\$ 49,999$ & 1.14 & $0.95-1.37$ & $P=0.15$ \\
\hline & $\$ 50,000-\$ 99,999$ & 1.06 & $0.95-1.19$ & $P=0.29$ \\
\hline & $\geq \$ 100,000$ & Ref & - & - \\
\hline
\end{tabular}

\section{Table 2}

Multivariable logistic regression of unread messages, clustered by patient, exclusive of "outreach" messages. 


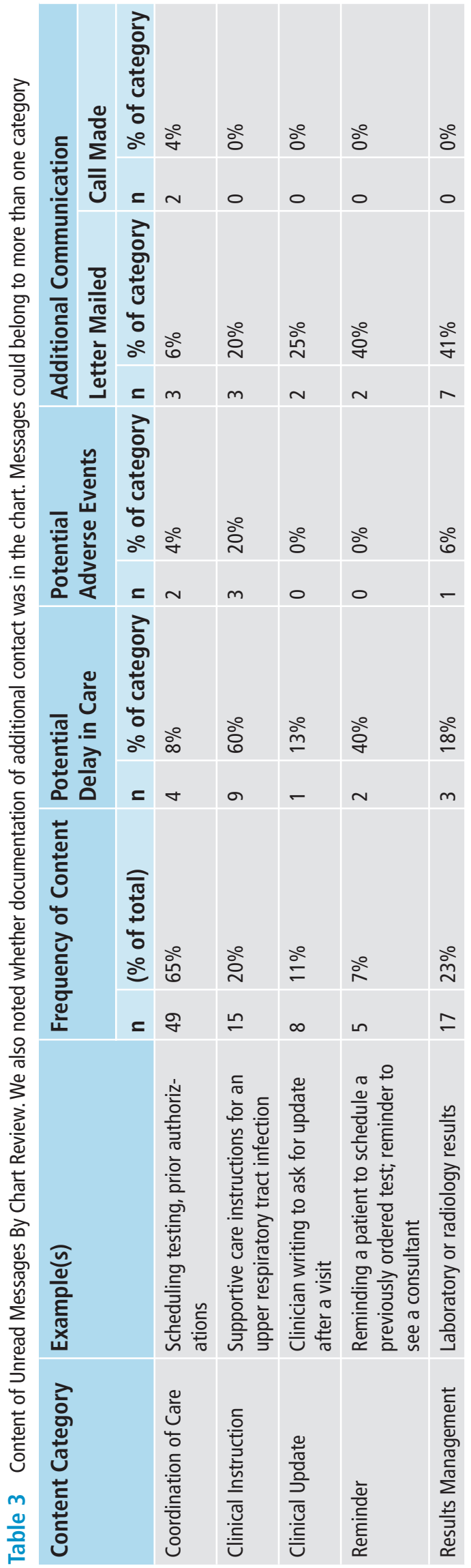




\section{References}

1. Slack WV. A 67-year-old man who e-mails his physician. JAMA 2004; 292(18): 2255-2261.

2. Adler KG. Web portals in primary care: an evaluation of patient readiness and willingness to pay for online services. J Med Internet Res 2006; 8(4): e26.

3. White CB, Moyer CA, Stern DT, and Katz SJ. A content analysis of e-mail communication between patients and their providers: patients get the message. J Am Med Inform Assoc 2004; 11(4): 260-267.

4. Anand SG, Feldman MJ, Geller DS, Bisbee A, Bauchner H. A content analysis of e-mail communication between primary care providers and parents. Pediatrics 2005; 115(5): 1283-1288.

5. Sittig DF. Results of a content analysis of electronic messages (email) sent between patients and their physicians. BMC Med Inform Decis Mak 2003; 3(1): 11.

6. Kane B, Sands DZ. Guidelines for the clinical use of electronic mail with patients. The AMIA Internet Working Group, Task Force on Guidelines for the Use of Clinic-Patient Electronic Mail. J Am Med Inform Assoc 1998; 5(1): 104-111.

7. North F, Crane SJ, Stroebel RJ, Cha SS, Edell ES, Tulledge-Scheitel SM. Patient-generated secure messages and eVisits on a patient portal: are patients at risk? J Am Med Inform Assoc 2013; 20(6): 1143-1149.

8. Byrne JM, Elliott S, Firek A. Initial experience with patient-clinician secure messaging at a VA medical center. J Am Med Inform Assoc 2009; 16(2): 267-270.

9. Bodenheimer T, Grumbach K. Electronic technology: a spark to revitalize primary care? JAMA 2003; 290(2): 259-264.

10. Margolius D, Bodenheimer T. Transforming primary care: from past practice to the practice of the future. Health Aff (Millwood) 2010; 29(5): 779-784.

11. Rohrer JE, North F, Angstman KB, Oberhelman SS, Meunier MR. Timely response to secure messages from primary care patients. Qual Manag Health Care 2013; 22(2): 161-166.

12. Halamka JD, Mandl KD, Tang PC. Early experiences with personal health records. J Am Med Inform Assoc 2008; 15(1): 1-7.

13. Weingart, S.N., D. Rind, Z. Tofias, and D.Z. Sands. Who uses the patient internet portal? The PatientSite experience. J Am Med Inform Assoc 2006; 13(1): 91-95.

14. Yamin CK et al. The digital divide in adoption and use of a personal health record. Arch Intern Med 2011; 171(6): 568-574.

15.US Census Bureau. American Community Survey. 2010 [cited 2013 Jan 1]. Available from: http://www. census.gov/acs/www/.

16. Slack WV. Claude Shannon and communication theory. MD Comput 1997; 14(4): 262-264.

17. Shannon CE, Weaver W. The Mathematical Theory of Communication. University of Illinois Press; 1971.

18. Osborn CY, et al. MyHealthAtVanderbilt: policies and procedures governing patient portal functionality. J Am Med Inform Assoc 2011; 18 (Suppl. 1): i18-i23.

19. Nazi KM. The personal health record paradox: health care professionals' perspectives and the information ecology of personal health record systems in organizational and clinical settings. J Med Internet Res 2013; 15(4): e70.

20. Bohmer RM. Managing the new primary care: the new skills that will be needed. Health Aff (Millwood). 2010; 29(5): 1010-1014.

21. Krist AH, et al. Electronic health record functionality needed to better support primary care. J Am Med Inform Assoc 2014 Jan 15. 